\begin{tabular}{|c|l|}
\hline Title & Temperature Independent Hole Mobility in Field-Effect Transistors Based on Liquid-Cry stall line Semiconductors \\
\hline Author(s) & Funahashi, Masahiro; Zhang, Fapei; Tamaoki, Nobuy uki \\
\hline Citation & $\begin{array}{l}\text { IEICE Transactions on Electronics, E94.C(11), 1720-1726 } \\
\text { https://doi.org/10.1587/transele.E94.C.1720 }\end{array}$ \\
\hline Issue Date & 2011-11-01 \\
\hline Doc URL & http://hdl.handle.net/2115/49110 \\
\hline Rights & Copyright $\odot 2011$ The Institute of Electronics, Information and Communication Engineers \\
\hline Type & article \\
\hline File Information & ToEe94c-11_1720-1726.pdf \\
\hline
\end{tabular}

Instructions for use 


\title{
Temperature-Independent Hole Mobility in Field-Effect Transistors Based on Liquid-Crystalline Semiconductors
}

\author{
Masahiro FUNAHASH ${ }^{\dagger a)}$, Fapei $\mathbb{Z H A N G}^{\dagger \dagger}$, and Nobuyuki TAMAOKI ${ }^{\dagger \dagger \dagger}$, Nonmembers
}

SUMMARY Thin-film transistors based on Liquid-crystalline phenylterthiophenes, 3-TTPPh-5 and 3-TTPPhF4-6 are fabricated with a spincoating method. The devices exhibit p-type operation with the mobility on the order of $10^{-2} \mathrm{~cm}^{2} \mathrm{~V}^{-1} \mathrm{~s}^{-1}$. The field-effect mobilities of the transistors using 3-TTPPh-5 and 3-TTPPhF4-6 are almost independent of the temperature above room temperature. In particular, the temperature range in which the mobility is constant is between 230 and $350 \mathrm{~K}$ for 3-TTPPh-5. key words: liquid-crystalline semiconductor, field-effect transistor, carrier transport, field-effect mobility, phenylterthiophene

\section{Introduction}

Liquid-crystalline (LC) semiconductors exhibit the high carrier mobility exceeding organic amorphous semiconductors as well as solution-processability [1]-[3]. Carrier transport properties in the LC phases have been studied by the timeof-flight method [4]-[6]. Macroscopic carrier mobilities in the bulk are revealed by this method, in contrast to the microscopic band mobilities determined by the pulse radiolysis time-resolved microwave conductivity [7]. The carrier mobilities are several orders of magnitude higher than those of organic amorphous semiconductors and exceeds $0.1 \mathrm{~cm}^{2} \mathrm{~V}^{-1} \mathrm{~s}^{-1}$ in the ordered smectic [8]-[10] and columnar phases [11] [13].

The other significant feature in the bulk carrier transport is temperature-independent mobility above room temperature. In a columnar phase of triphenylene dimer, the hole mobility is independent of the temperature above room temperature and decreases with a decrease in the temperature below it [5]. The same tendency is observed in the SmE phase of alkynylterthiophene derivatives both for holes and electrons. A mechanism based on the disorder formalism has been proposed to explain the temperature-independence of the carrier mobilities in the LC phases [5], [6]. This independence has been attributed to a small energetic disorder in the LC phases compared to the amorphous phases. In addition, the influence of the defects such as domain boundaries is small in the LC phases compared to crystal phases [14]. In the LC phases, the defects are more ambiguous due

\footnotetext{
Manuscript received February 25, 2011.

Manuscript revised June 6, 2011.

The author is with Kagawa University, Takamatsu-shi, 7610396 Japan.

The author is with Chinese Academy of Science, Hefei, 230031 P.R. China.

tti The author is with Hokkaido University, Sapporo-shi, 0010020 Japan.

a) E-mail: m-funa@eng.kagawa-u.ac.jp DOI: $10.1587 /$ transele.E94.C.1720
}

to molecular thermal movement and fluctuation than in the crystal phases.

Field-effect transistors (FETs) based on LC semiconductors such as oligothiophene [10], [15]-[17] and hexabenzocoronene derivatives have been reported recently [18]. Phenylterthiophene derivatives can produce LC thin films on silicon and polymer substrates [10], [19]-[21] by the spincoating method. However, the temperature-dependence of the field-effect mobilities in the thin film states of the LC semiconductors has not been studied so far. The typical value of the thickness of the active layers in the FETs is several ten nanometers. The carrier transport in the thin films should be different from those of the bulk LC states.

In FETs based on vacuum-deposited aromatic polycrystalline thin films, the defect density and impurity contamination determine the temperature-dependence of the carrier mobilities [22]. Temperature-independent hole mobility was observed in the FETs using vacuum-deposited thin films of pentacene with the high quality [23]. In this study, we report LC thin films of phenylterthiophene and tetrafluorophenylterthiophene derivatives as well as the fabrication of FETs based on these LC compounds. And we also report the temperature-independent field-effect hole mobilities in the LC FETs based on these LC compounds.

\section{Experiments}

\subsection{Synthesis of LC Semiconductors}

Oligothiophene derivatives are widely studied as active layers of electroluminescence devices and FETs [24]. However their solubilities in organic solvents are too low for the solution process. Dialkylterthiophene and dialkylquaterthiophene derivatives exhibit smectic phases, and efficient ambipolar carrier transport has been observed [25], [26]. However they crystallize around room temperature resulting in inhomogeneous thin films with the solution process.

We have already reported that 5-alkyl-5"-alkynyl2, 2':5', 2'-terthiophene and 5-alkyl-5'"-alkynyl-2, 2':5', $2 ": 5 ", 2$ "'-quaterthiophene exhibit ordered smectic phases around room temperature. Asymmetric structures of these compounds should inhibit the crystallization and result in the appearance of the metastable LC phases at room temperature [6], [9].

Other asymmetrically substituted terthiophene derivatives which bear alkyl and phenyl or tetrafluorophenyl substituents also exhibit the ordered smectic phases around 

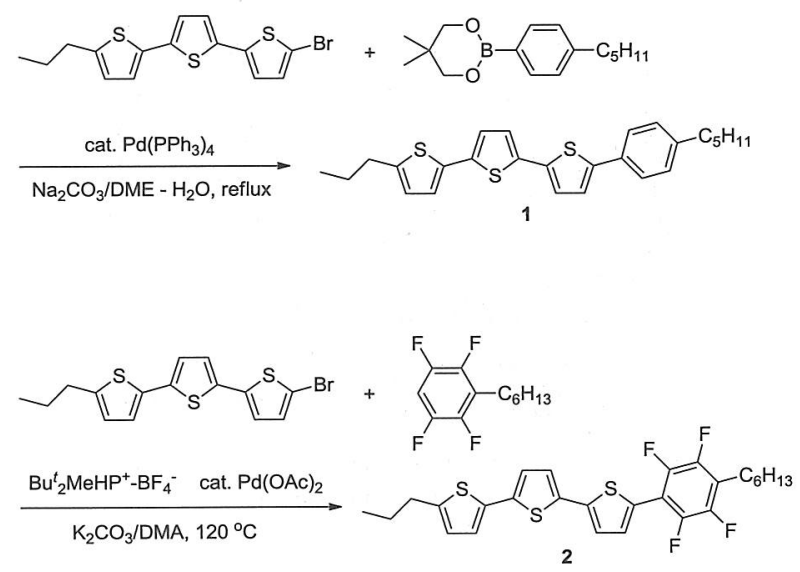

Scheme 1 Synthetic routes of LC semiconductors 1 and 2.

Table 1 Phase transition temperatures of LC semiconductors 1 and 2. OrdSm: ordered smectic phase; N: nematic phase; Iso: isotropic phase.

\begin{tabular}{llllll}
\hline Compound & Phase transition temperature & \\
\hline 3-TTPPh-5 (1) & OrdSm $202{ }^{\circ} \mathrm{C}$ & $\mathrm{N}$ & $217{ }^{\circ} \mathrm{C}$ & Iso \\
3-TTPPhF4-6 (2) & OrdSm $112{ }^{\circ} \mathrm{C}$ & $\mathrm{N}$ & $142{ }^{\circ} \mathrm{C}$ & Iso \\
\hline
\end{tabular}

room temperature. 5-propyl-5"'-(4-pentylphenyl)-2, 2':5', 2"-terthiophene (1) (3-TTPPh-5) and 5-propyl-5"-(2, 3, 5, 6-tetrafluoro-4-hexylphenyl)-2, 2':5', 2"'-terthiophene (2) (3-TTPPhF4-6) were synthesized by the $\mathrm{Pd}(0)$-catalyzed coupling reactions as shown in Scheme 1 . These compounds exhibit the ordered smectic phases at room temperature and are soluble in conventional organic solvents such as toluene, xylene, and tetrahydrofurane [10], [20]. The phase transition temperatures are summarized in Table 1.

\subsection{Preparation of the FETs on $\mathrm{SiO}_{2} / \mathrm{Si}$ Substrates}

FETs using the LC semiconductors were fabricated on $\mathrm{Si} / \mathrm{SiO}_{2}$ substrates. The chlorobenzene solution $(0.6 \mathrm{wt} \%)$ of compound $\mathbb{1}$ or 2 was spun on a $\mathrm{Si} / \mathrm{SiO}_{2}$ substrate at $1500 \mathrm{rpm}$ for $25 \mathrm{~s}$, producing homogeneous LC thin films with the thickness of $50 \mathrm{~nm}$. On the LC thin films, Au source and drain electrodes were vacuum-deposited through a shadow mask. The better performance is obtained when the surface of the $\mathrm{SiO}_{2}$ layer is treated with a silane coupling reagent [19].

\section{Results and Discussion}

\subsection{Mesomorphic Behaviors of LC Semiconductors}

3-TTPPh-5 (1) exhibited an ordered smectic phase around room temperature and did not crystallize even when it was cooled to $-50^{\circ} \mathrm{C}$. In the ordered smectic phase, the X-ray diffraction reveals that a long range order exists within the smectic layers and the LC molecules tilted towards the normal of the smectic layers at an angle of $57^{\circ}$. This result indicates that this smectic phase has a closer packing structure

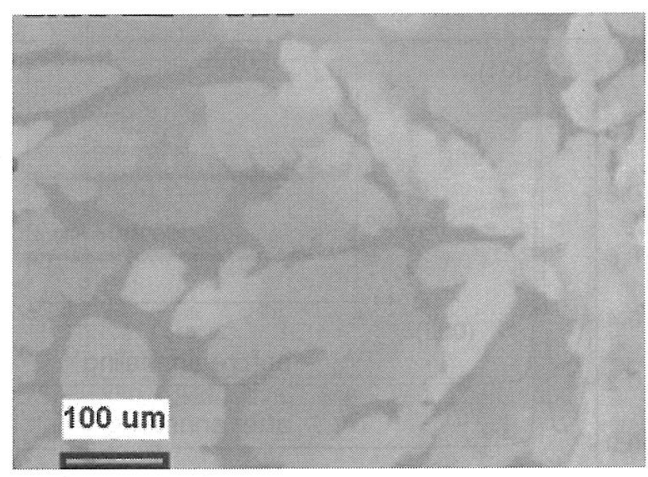

Fig. 1 Polarizing optical micrographic image of a LC thin film of 3TTPPh-5 (1) produced by a spin-coating method.

that is favorable for fast carrier transport.

3-TTPPhF4-6 (2) exhibits an enantiotropic nematic $(\mathrm{N})$ and an ordered smectic phases. When the ordered smectic phase was cooled to $-50^{\circ} \mathrm{C}$, no glass transition was observed. The X-ray diffraction of the ordered smectic phase of compound 2 indicates a crystal-like three-dimensional structure. However, this phase exhibits fluidity above 100 ${ }^{\circ} \mathrm{C}$ on heating, below the phase transition to the nematic phase, indicating that this is a mesophase with a freedom of molecular thermal movement.

The phase transition temperatures of compound 2 were lowered by ca. $50^{\circ} \mathrm{C}$ as compared to the non-fluorinated counterpart $\mathbf{1}$, as shown in Table 1.

\subsection{Characterization of the LC Thin Films of 3-TTPPh-5} (1)

Figure 1 shows a polarizing optical micrograph of the LC thin film of 3-TTPPh-5 (1) produced by the spin-coating method. The thin film comprised of optically anisotropic domains of which sizes were on the order of several ten micrometers.

As shown in Fig. 2(a), a sharp diffraction peak at the angle $2 \theta=3.9^{\circ}$ was observed in the out-of-plane X-ray diffraction measurement. The layer spacing was almost the same as that of the bulk smectic phase. In the wide angle region of the in-plane diffraction, only a broad halo around $21^{\circ}$ was observed in the as-deposited film (Fig. 2(b)). This $\mathrm{X}$-ray diffraction curves indicate that this thin film has a layer structure but not a long-range order within layers. After thermal annealing, the wide angle halo changed to a clear peak, indicating a long-range order within the smectic layers.

The atomic force microscopy (AFM) observation also exhibits the enhancement of the ordering of the LC molecules by the thermal treatment. The surface of the asdeposited film was not flat but showed a roughness on the order of several nanometers, as shown in the photographs at the top of Fig. 3(a). However, the thermal annealing of the thin films at $120^{\circ} \mathrm{C}$ for $15 \mathrm{~min}$, the surface morphology was remarkably changed as shown in Fig. 3(b). The surface was completely flat on the molecular scale. This indicates that 


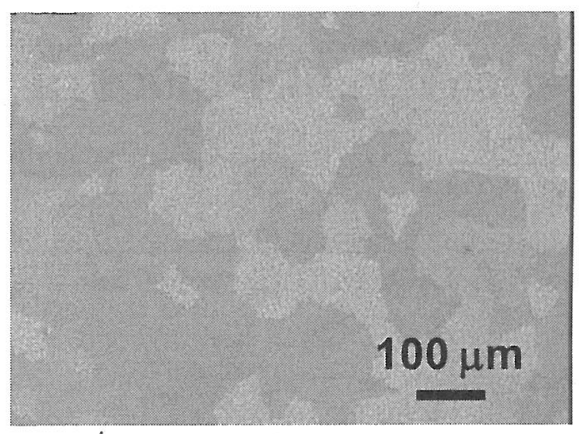

(a)
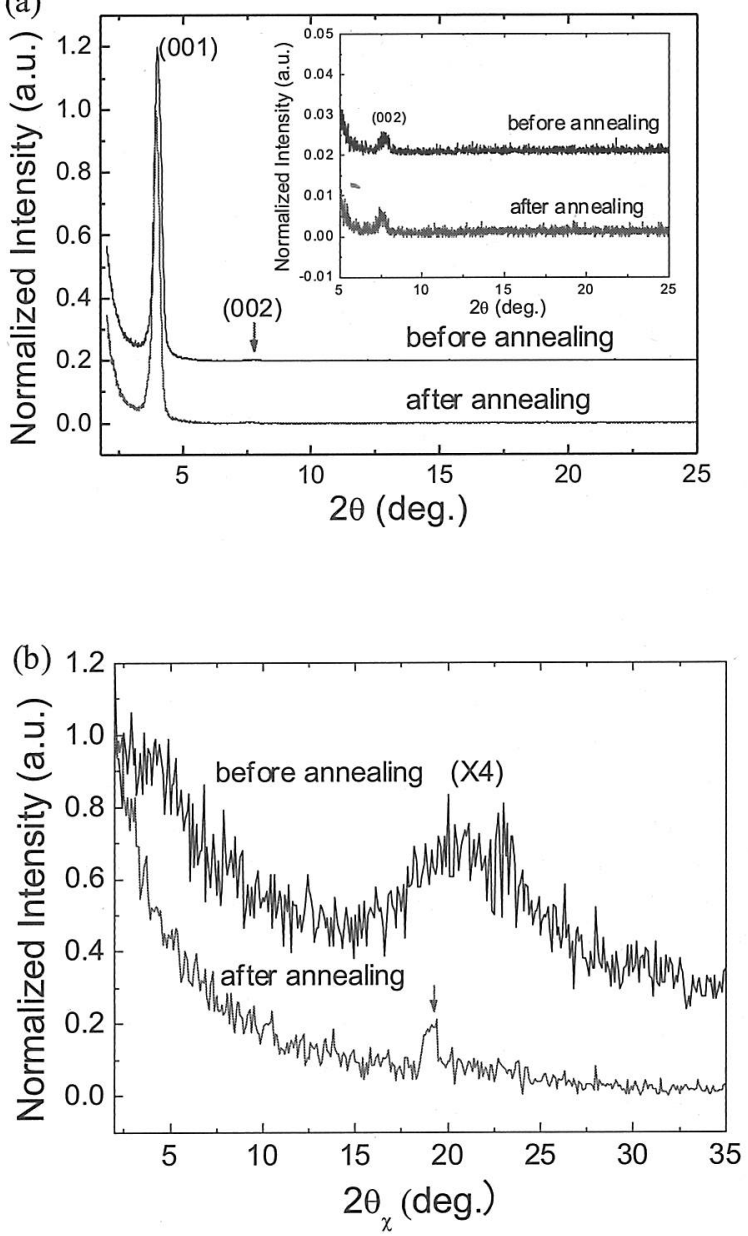

Fig. 2 X-ray diffraction patterns of a thin film of 3-TTPPh-5 (1) measured in (a) out-of-plane and (b) in-plane configurations. The inset in (a) is an extension of the curves in the region between 5 and 25 degrees. The curve in the in-plane configuration (b) is magnified by a factor of 4 .

(a)

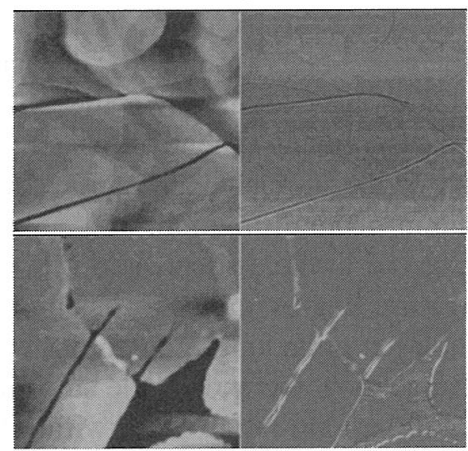

Fig. 3 AFM images of the thin film (a) before and (b) after annealing at $120^{\circ} \mathrm{C} / 15 \mathrm{~min}$. Left: topographical image, Right: phase image.

the domain has an ordered structure and low defect density. The domain size was $50-100 \mu \mathrm{m}$, which is larger than the channel length $(20-30 \mu \mathrm{m})$ of the normal FETs. It should be noted that the freedom of molecular movement in the LC phase should promote the reorganization of molecular alignment in the thin films, resulting in the formation of high-
Fig. 4 Polarizing optical micrographic image of a LC thin film of 3TTPPhF4-6 (2) produced by a spin-coating method.

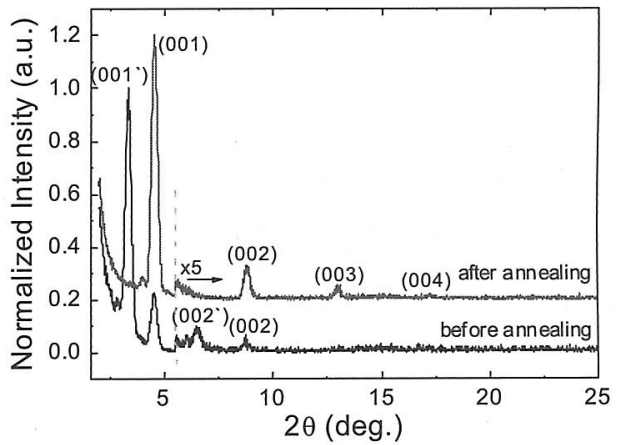

Fig. 5 X-ray diffraction patterns of a thin film of 3-TTPPhF4-6 (2) before and after the thermal treatment at $85^{\circ} \mathrm{C}$ for $10 \mathrm{~min}$.

quality thin films.

\subsection{Characterization of the LC Thin Films of 3-TTPPhF4- $6(2)$}

Figure 4 shows a polarizing optical micrograph of a thin film for 3-TTPPhF4-6 (2) after thermal annealing at $85^{\circ} \mathrm{C}$ for $10 \mathrm{~min}$. During the thermal treatment, a fine threaded texture changed to a mosaic texture. The domain sizes exceed several hundred micrometers. In these LC thin films of compound 2 , the enhancement of the reorientation of the $\mathrm{LC}$ molecules with the thermal treatment was also observed.

In the X-ray diffraction patterns as shown in Fig. 5, the as-deposited films of compound $\mathbf{2}$ show two distinct series of intense peaks, suggesting the presence of two different LC phases. After the thermal annealing, the diffraction peaks belonging to one series disappeared completely and the intensity of the peaks in the other series was considerably enhanced. Under the condition of spin-coating, a metastable LC phase should appear, due to the fast evaporation of the solvent. These two LC phases have layer structures and the tilt angles of the LC molecules should be different between the LC phases. The interlayer spacing was calculated as $2.1 \mathrm{~nm}$ after thermal annealing, in agreement with the step height of the molecular layers measured by AFM. This indicates that the smectic layers in the film are parallel to the substrate surface. 

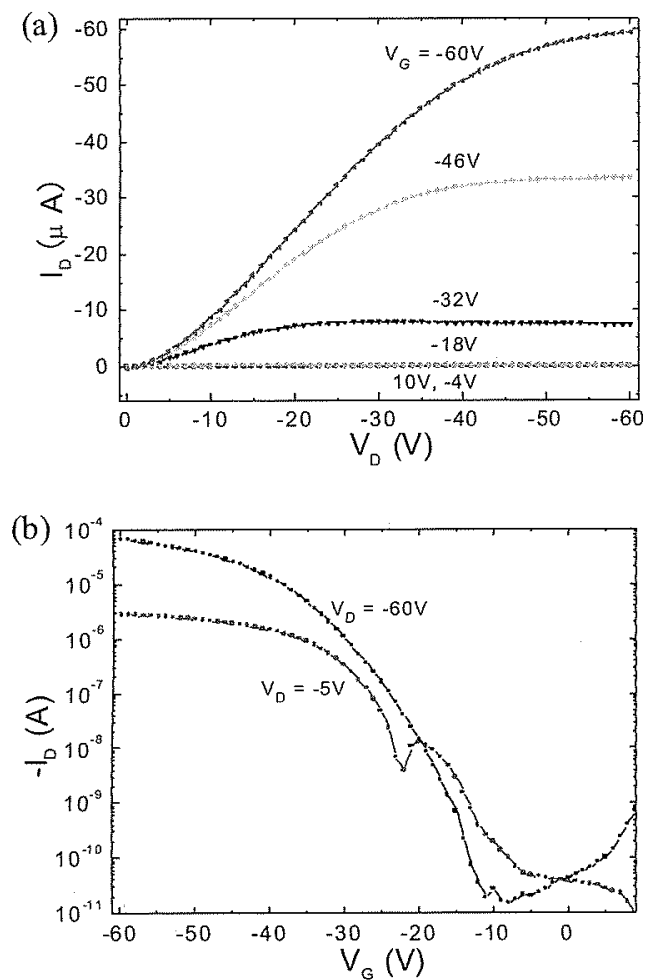

Fig. 6 (a) Output and (b) transfer characteristics of the FET based on the LC thin film of 3-TTPPh-5 (1). The channel length and film thickness were $20 \mu \mathrm{m}$ and $65 \mathrm{~nm}$, respectively.

\subsection{Characteristics of the Field-Effect Transistors Using 3-} TTPPh-5 (1) and 3-TTP-PhF4-6 (2)

The FETs fabricated based on 3-TTPPh-5 (1) showed p-type operation. When the $\mathrm{SiO}_{2}$ surface was treated by octyltrichlorosilane, the best performance was obtained. Figure 6 exhibits the out-put and transfer characteristics of the device. The mobility of $4.2 \times 10^{-2} \mathrm{~cm}^{2} \mathrm{~V}^{-1} \mathrm{~s}^{-1}$ and the on/off ratio of more than $10^{6}$ are achieved after the thermal treatment at $105^{\circ} \mathrm{C}$ for $5 \mathrm{~min}$. In these devices, the smectic layers are aligned parallel to the $\mathrm{SiO}_{2}$ surface and the holes are transported within the layers as a consequence. The value is comparable to the hole mobility obtained from the TOF measurement [20], to represent an upper limit to the mobility of the FETs.

Figure 7 shows the temperature-dependence of the field-effect mobility for holes and the threshold voltage in the FET based on compound 1. Between 200 and $350 \mathrm{~K}$, the hole mobility is independent of the temperature. Below $200 \mathrm{~K}$, the hole mobility decreases remarkably with a decrease in the temperature. In this low temperature region, a few cleavages were formed in the LC thin films because of the volume shrinkage. Above $350 \mathrm{~K}$, the mobility also decreases gradually with an increase in the temperature. This should be originated from the increase in the disorder, due to the thermal movement of the LC molecules. The threshold voltage $V_{T}$ shifts to negative direction with a decrease
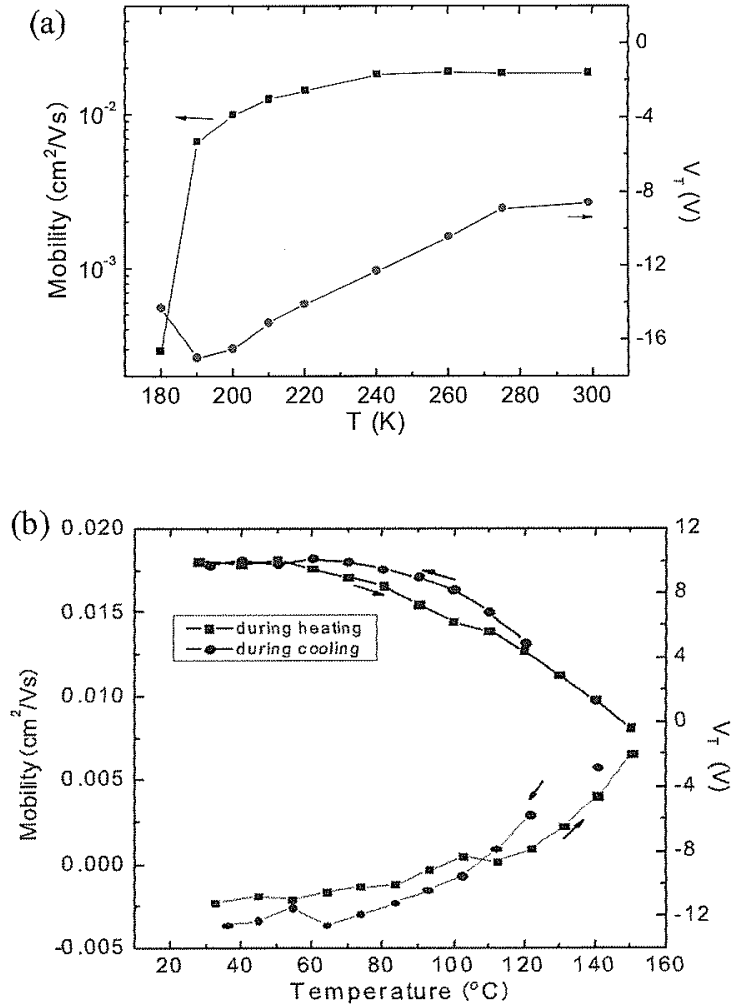

Fig. 7 Hole mobility and threshold voltage of the FET using 3-TTPPh$5(1)$ as a function of the temperature in (a) below and (b) above room temperature.

in the temperature in the both regions. In the low temperature region, this behavior should be attributed to the hole trapping on the $\mathrm{SiO}_{2}$ surfaces. Above room temperature, thermal movement of the LC molecules should affect on the detrapping of the holes at the interface. In the field-effect mobility and the threshold voltage exhibit a slight hysteresis for the heating and cooling processes. This should be attributed to the change of the molecular ordering depending on the temperature.

The field-effect mobility decreased to $2 \times 10^{-2} \mathrm{~cm}^{2} \mathrm{~V}^{-1}$ $\mathrm{s}^{-1}$ when the channel length was increased to $50 \mu \mathrm{m}$. This indicates that the FET characteristics are determined mainly by the hole transport in the active layer rather than contact resistance.

In the LC thin films of 3-TTPPh-5 (1) in the FETs, the size of the LC domains exceeds the channel lengths. Therefore, the holes can drift between the source and drain electrodes without encountering the domain boundaries on which localized states are formed. In vacuum-deposited molecular crystals, grain size is smaller than the channel length. The device performance is strongly affected from the crystal growth condition and depends on the temperature as a result.

Figure 8(a) shows the $I_{D}-V_{D}$ characteristics for FETs of 3-TTPPhF4-6 (2) measured in ambient air. The devices exhibit a typical p-type operation. The hole mobility is calculated as $2.7 \times 10^{-2} \mathrm{~cm}^{2} \mathrm{~V}^{-1} \mathrm{~s}^{-1}$ in a saturation region. The 


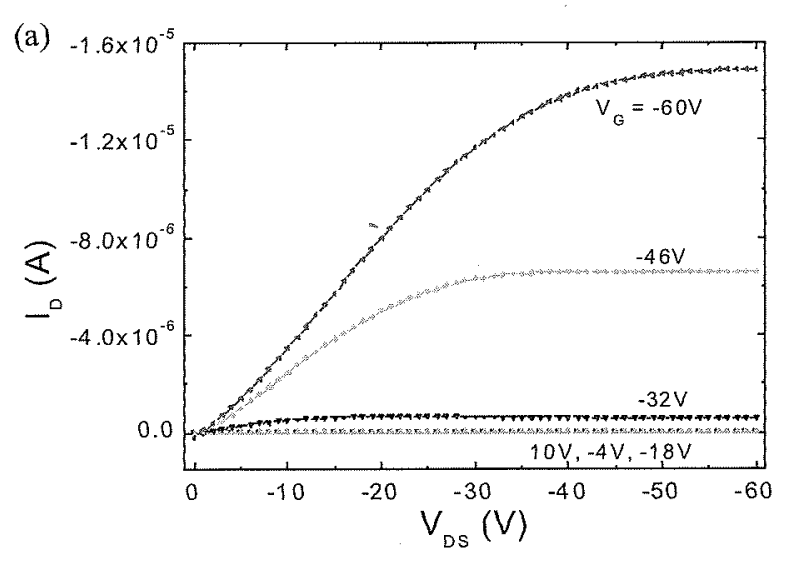

(b)

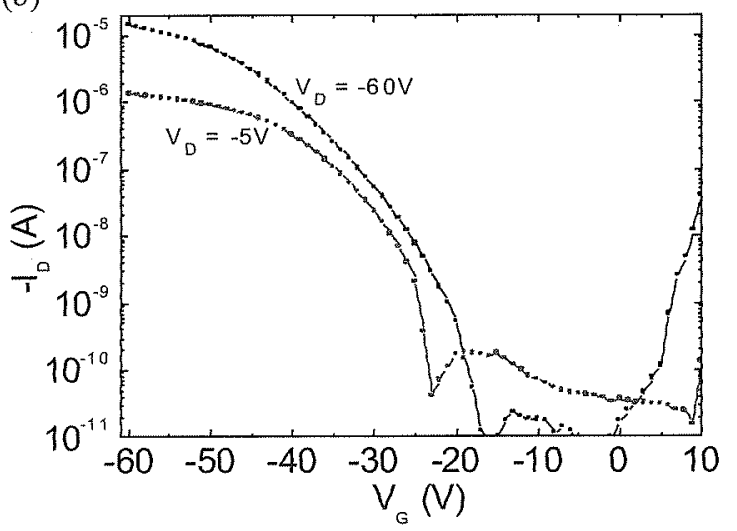

Fig. 8 (a) Output and (b) transfer characteristics of the FET based on the LC thin film of 3-TTPPhF4-6 (2). The channel length and film thickness were $20 \mu \mathrm{m}$ and $50 \mathrm{~nm}$, respectively.

current on/off ratio is more than $10^{6}$. Such a value is close to that obtained from the TOF measurement in the bulk state $\left(7 \times 10^{-2} \mathrm{~cm}^{2} \mathrm{~V}^{-1} \mathrm{~s}^{-1}\right.$ at $\left.50^{\circ} \mathrm{C}\right)$ [19]. The mobility is also comparable to that of the FETs of non-fluorinated compound 1. Moreover, an enhanced stability of operation in ambient air is expected for the FETs of compound 2 . The strong electron withdrawing characteristics of the tetrafluorophenyl group, which raise the oxidation potential of the aromatic core to make the material less susceptible to air oxidation.

Figure 9 shows the temperature-dependence of the hole mobility and the threshold voltage of the devices using compound 2 . The hole mobility is almost independent of the temperature between 27 and $80^{\circ} \mathrm{C}$. This should be attributed to the formation of highly ordered LC domains with the size larger than the channel length $(20-30 \mu \mathrm{m})$ of the FETs. The holes can be transported in the channel area without being trapped. In the FETs based on compound 2, the temperature-dependence of the threshold voltage is also small. This should be attributed to the low density or shallow depth of the localized states on the dielectric surface.

The most significant characteristic of these LC-based FETs is the temperature-independent carrier mobility. Bulk carrier transport characteristics have been studied by the time-of-flight technique. In the columnar and smectic

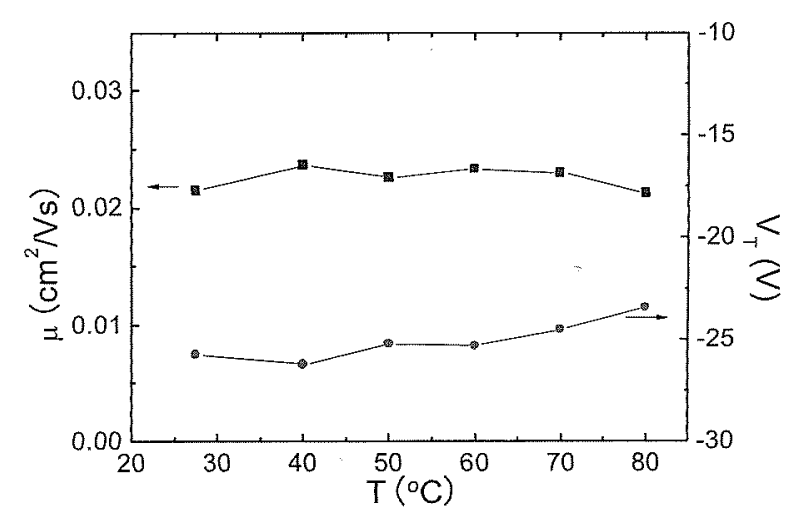

Fig.9 Hole mobility $\mu$ (filled square) and threshold voltage $\mathrm{V}_{\mathrm{T}}$ (filled circle) of the FET using 3-TTPPhF4-6 (2) as a function of the temperature.

phases, temperature-independent carrier mobilities have been observed above critical temperatures [5], [6]. In the case of LC alkynylterthiophene, the temperature is $-20^{\circ} \mathrm{C}$. Below the temperature, the carrier mobilities usually depend upon the temperature and the electric field. The dependences are explained by the Gaussian disorder model which was proposed for carrier transports of amorphous organic semiconductors. This model assumes hopping transport between molecular orbitals and the Gaussian distribution of their energy levels. In this model, photo-generated carriers are thermally relaxed and activated by the heat and the electric field, leading to the temperature and the field-dependent carrier mobility.

The width of the distribution is narrower in the LC phases than in the amorphous materials, due to the molecular order in the LC phases. In the high temperature regions, most of the photo-generated carriers should be located close to the transport level, resulting in the temperatureindependent mobility [5], [6].

There is a possibility that coincidental compensation of the thermal activation effect in the carrier hopping process for the thermal fluctuation of the LC phase structure produces the apparent temperature-independent mobilities. In fact, a complicated dependence of the hole mobility on the temperature was also reported for a columnar phase consisting of a triphenylene dimer [27]. In this case, the hole mobility exhibits a maximum value as a result of an imperfect balance between the two effects. However, the ordered smectic phases exhibit fluidity just below the phase transition temperature to the nematic or isotropic phases. In general, ordered smectic and columnar structures determined by the X-ray diffraction are not so perturbed by the thermal effect below room temperature [5], [6]. The thermal fluctuation of LC molecules should not cancel the temperaturedependence of the carrier mobility caused by thermal activation of the carrier hopping in a wide temperature region below room temperature.

In this study, the temperature-independent mobility is also observed over a wide temperature range in the LCbased FETs in which the carriers move within the twodimensional interface areas unlike the three-dimensional 
bulk states. This result indicates that the ordered structure with the small energetic disorder and the low defect density should be formed not only in the bulk but also at the interface area between the active layer and the gate insulator.

The temperature-dependent carrier mobilities have been observed in the FETs based on high quality polycrystalline films [28] and rubrene single crystals [29], [30]. In these cases, carrier mobilities exceed $1 \mathrm{~cm}^{2} \mathrm{~V}^{-1} \mathrm{~s}^{-1}$, and the carrier transport process is band mechanism. The balance between the effects of phonon scattering and thermal activation from shallow traps results in the apparent temperatureindependent mobilities.

In contrast, the carrier mobilities of LC semiconductors are on the order of $10^{-1} \mathrm{~cm}^{2} \mathrm{~V}^{-1} \mathrm{~s}^{-1}$ at best. This value is one order of magnitude lower than those of organic crystalline semiconductors. The carrier transport processes are based on hopping mechanism rather than band scheme. As shown in Fig. 7, the decrease in the hole mobility above $80^{\circ} \mathrm{C}$ should be attributed to the increase of the structural disorder caused by thermal movement of the LC molecules. However, below the temperature, the thermal fluctuation should be suppressed and the LC structure is stable. The temperature-independent mobility should be ascribed to the small energetic disorder and small influence from the domain boundaries in the LC phases comparing with the amorphous organic semiconductors. The detailed study on the mechanism is in progress.

Comparing to organic crystalline semiconductors, large domains whose sizes exceed FET channel lengths can be formed with quite simple processes. For the FETs based on crystalline thin films of aromatic compounds, a precise control of the crystal growth is indispensable under a vacuum condition in order to obtain the temperatureindependent carrier mobilities. In the LC semiconductors, the LC materials are purified by a column chromatography and recrystallization, and the devices are fabricated by the spin-coating method. The material purifications and device fabrications are carried out under ambient conditions.

\section{Conclusion}

FETs with the good performance were fabricated using LC semiconductors, 3-TTPPh-5 (1) and 3-TTPPhF4-6 (2) by the spin-coating method. They exhibit p-type operation with the field-effect mobility on the order of $10^{-2} \mathrm{~cm}^{2} \mathrm{~V}^{-1} \mathrm{~s}^{-1}$ and the on/off ratio of $10^{6}$. It should be noted that the devices are operated in the LC state, in which large domains are formed in the channel areas. The domain sizes exceed several tens micrometers and the holes can move in the channel areas without being trapped. In consequence, the hole mobilities of the FETs for both LC semiconductors are almost independent of the temperature.

\section{Acknowledgments}

This study was financially supported by the NEDO Industrial Technology Research Grant, Association of New
Chemistry, Sumitomo foundation, the Japan Security Scholarship Foundation, a-step from Japan Science and Technology Agency (JST), Grant-in-Aid for Scientific Research on Innovative Areas (Coordination Programming, no. 22108506) from Ministry of Education, Culture, Sports, Science and Technology (MEXT), and a Grant-in-Aid for Scientific Research (B) (no. 22350080) from the Japan Society for the Promotion of Science (JSPS). We thank Dr. H. Minamikawa and the Nano-Processing Facility in National Institute of Advanced Industrial Science and Technology (AIST) for providing the XRD apparatus. We also thank Dr. M. Chikamatsu and Dr. R. Azumi of Photonics Research Institute of AIST for their fruitful advice on device characterization and Prof. T. Kato for the valuable discussion on the material design. We also thank Mitsui Chemicals for providing the polyimide sheets.

\section{References}

[1] M. O'Neill and S.M. Kelly, "Ordered materials for electronics and photonics," Adv. Mater., vol.23, pp.566-584, 2011

[2] Y. Shimizu, K. Oikawa, K. Nakayama, and D. Guillon, "Mesophase semiconductors in field effect transistors," J. Mater. Chem., vol.17, pp.4223-4229, 2007.

[3] M. Funahashi, "Development of liquid-crystalline semiconductors with high carrier mobilities and their application to thin-film transistors," Polymer J., vol.41, pp.459-469, 2009.

[4] D. Adam, F. Closs, T. Frey, D. Funhoff, D. Haarer, H. Ringsdorf, P. Schuhmacher, and K. Siemensmeyer, "Transient photoconductivity in a discotic liquid crystal," Phys. Rev. Lett., vol.70, pp.457-460, 1993.

[5] I. Bleyl, C. Erdelen, H.-W. Schmidt, and D. Haarer, "Onedimensional hopping transport in a columnar discotic liquidcrystalline glass," Philos. Mag., vol.B79, pp.463-475, 1999.

[6] M. Funahashi, F. Zhang, N. Tamaoki, and J. Hanna, "Ambipolar transport in the smectic E phase of 2-propyl-5"-hexynylterthiophene derivative over a wide temperature range," Chem Phys Chem., vol.9, pp.1456-1473, 2008.

[7] A.M. van de Craats, J.M. Warman, A. Fechtenkötter, J.D. Brand, M.A. Harbison, and K. Müllen, "Record charge carrier mobility in a room-temperature discotic liquid-crystalline derivative of hexabenzocoronene," Adv. Mater., vol.11, pp.1469-1471, 1999.

[8] K. Oikawa, H. Monobe, J. Takahashi, K. Tsuchiya, B. Heinrich, D Guillon, and Y. Shimizu, "A novel calamitic mesophase semiconductor with the fastest mobility of charged carriers: 1,4-di(5'-octyl2'-thienyl)benzene," Chem. Commun., pp.5337-5339, 2005.

[9] M. Funahashi and J. Hanna, "High carrier mobility up to $0.1 \mathrm{~cm}^{2} \mathrm{~V}^{-1} \mathrm{~s}^{-1}$ at ambient temperatures in thiophene-based liquid crystals," Adv. Mater., vol.17, pp.594-598, 2005.

[10] M. Funahashi, F. Zhang, and N. Tamaoki, "High ambipolar mobility in a highly ordered smectic phase of a dialkylphenylterthiophene derivative that can be applied to solution-processed organic fieldeffect transistors," Adv. Mater., vol.19, pp.353-358, 2007.

[11] D. Adam, P. Schuhmacher, J. Simmerer, L. Häußling, K Siemensmeyer, K. Etzbach, H. Ringsdorf, and D. Haarer, "Fast photoconduction in the highly ordered columnar phase of a discotic liquid crystal," Nature, vol.371, pp.141-143, 1994.

[12] A.M. van de Craats and J.M. Warman, "The core-size effect on the mobility of charge in discotic liquid crystalline materials," Adv Mater., vol.13, pp.130-133, 2001.

[13] H. Ino, J. Hanna, R.J. Bushby, B. Movaghar, B.J. Whitaker, and M.J. Cook, "Very high time-of-flight mobility in the columnar phases of a discotic liquid crystal," Appl. Phys. Lett., vol.87, $132102,2005$. 


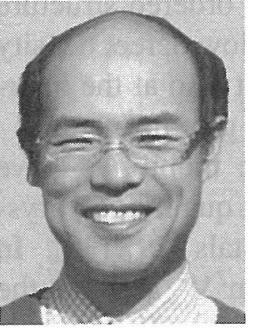

Masahiro Funahashi received Ph.D. from Tokyo Institute of Technology in 1999. He had been a research associate of Tokyo Institute of Technology during 1994-2005, and a researcher of AIST during 2005-2007. He promoted to an associate professor of the University of Tokyo in 2007. He is a professor of Kagawa University since 2010.

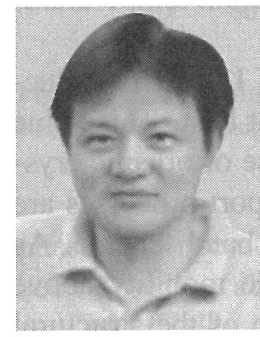

Fapei Zhang received Ph.D. from Technische Universität, Dresden in 2003. After working in Institute for Molecular Science and Osaka University, he joined to the LC FET project in AIST in 2006. Now he is a senior researcher of High Magnetic Field Laboratory, Chinese Academy of Science.

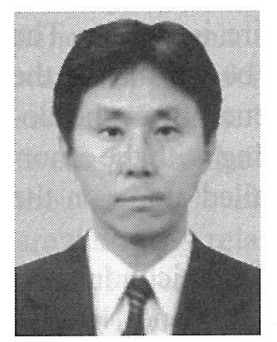

Nobuyuki Tamaoki received Ph.D. from Chiba University in 1992. In 1992, he joined the Research Institute for Polymers and Textiles. He was a group leader of AIST from 2000 to 2008. In 2008, he moved to Research Institute for Electronic Science, Hokkaido University as a full professor. 